\begin{aligned} Journal homepage: $\underline{w w}$. eurjehem.com \\ \hline\end{aligned}

\title{
Stereoselective synthesis of (-) Cephalosporolide D
}

\author{
Karna Ji Harkala a, Laxminarayana Eppakayala ${ }^{a, *}$ and Thirumala Chary Maringanti b \\ a Department of Physics and Chemistry, Mahatma Gandhi Institute of Technology, Chaitanya Bharati, Gandipet, Hyderabad-500075, India \\ b Department of Chemistry, Jawaharlal Nehru Technological University Hyderabad College of Engineering, Nachupally, Karimnagar-505501, India
}

${ }^{*}$ Corresponding author at: Department of Physics and Chemistry, Mahatma Gandhi Institute of Technology, Chaitanya Bharati, Gandipet, Hyderabad-500075, India. Tel.: +91.998.9291441. Fax: +91.40.24193067. E-mail address: elxnkits@yahoo.co.in (L. Eppakayala).

\section{ARTICLE INFORMATION}

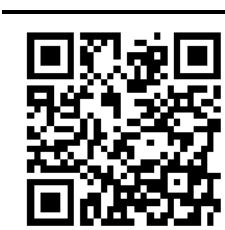

DOI: $10.5155 /$ eurjchem.5.1.127-132.1002

Received: 15 December 2013

Received in revised form: 16 February 2014

Accepted: 01 March 2014

Online: 31 March 2014

\section{KEYWORDS}

$\alpha$-Aminoxylation

Wittig olefination

Catalytic hydrogenation

Epoxidation with mCPBA

Yamaguchi macrolactonisation

Hydrolytic kinetic resolution of epoxide

\section{Introduction}

The macrolides, which contain medium-sized ring systems, have attracted considerable attention from synthetic chemists due to their interesting biological properties [1-4].

Cephalosporolide D (3), a eight-membered lactone, isolated from fermentation fungus, Cephalosporiumaphidicola, along with four other cephalosporolides (B, C and E-G) (Figure 1) [5,6]. Among them, cephalosporolide D (3) is an eightmembered lactone, cephalosporolides B, C and G are tenmembered ring lactones and the others (cephalosporolide $\mathrm{E}$ and F) are spiroketal lactones. Because of their fascinating structural features and interesting biological properties, cephalosporolides have solicited considerable interest among organic chemists [7-12].

In accordance with our interest in the natural product synthesis, herein we report an alternative total synthesis of Cephalosporolide D starting from commercially available starting materials.

\section{Experimental}

\subsection{Instrumentation}

Solvents were dried over standard drying agents on freshly distilled prior to use. Chemicals were purchased and used without further purification. All column chromatographic separations were performed using silica gel (60-120 mesh) Organic solutions were dried over anhydrous $\mathrm{Na}_{2} \mathrm{SO}_{4}$ and concentrated below $40{ }^{\circ} \mathrm{C}$ in vacuum. ${ }^{1} \mathrm{H}$ NMR spectra were acquired at $300 \mathrm{MHz}$, while, ${ }^{13} \mathrm{C}$ NMR at $75 \mathrm{MHz}$ with TMS as internal standard for solutions in $\mathrm{CDCl}_{3} . J$ values were given in Hz. IR spectra were recorded on FTIR spectrophotometer with $\mathrm{NaCl}$ optics. Optical rotations were measured on digital polarimeter at $25{ }^{\circ} \mathrm{C}$. Mass spectra were recorded on direct inlet system or LC by MSD trap SL, the HRMS data were obtained using Q-TOF mass spectrometry.<smiles>CCC(C)CC(=O)/C=C\C(=O)CC(=O)O</smiles>

(+)-Cephalosporolide B (1)<smiles>COC(C)CCC(=O)CC(O)C(O)CC(=O)O</smiles>

(+)-Cephalosporolide C (2)

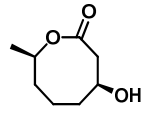

$(-)$ Cephalosporolide D (3)

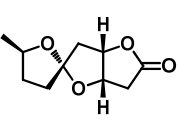

(+)-Cephalosporolide E (4)

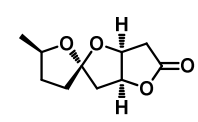

Cephalosporolide $\mathrm{F}$ (5)<smiles>COC(C)CCC(=O)C[C@@H](O)[C@H](O)CC(=O)O</smiles>

Cephalosporolide G (6)
Figure 1. Structures of cephalosporolides. 

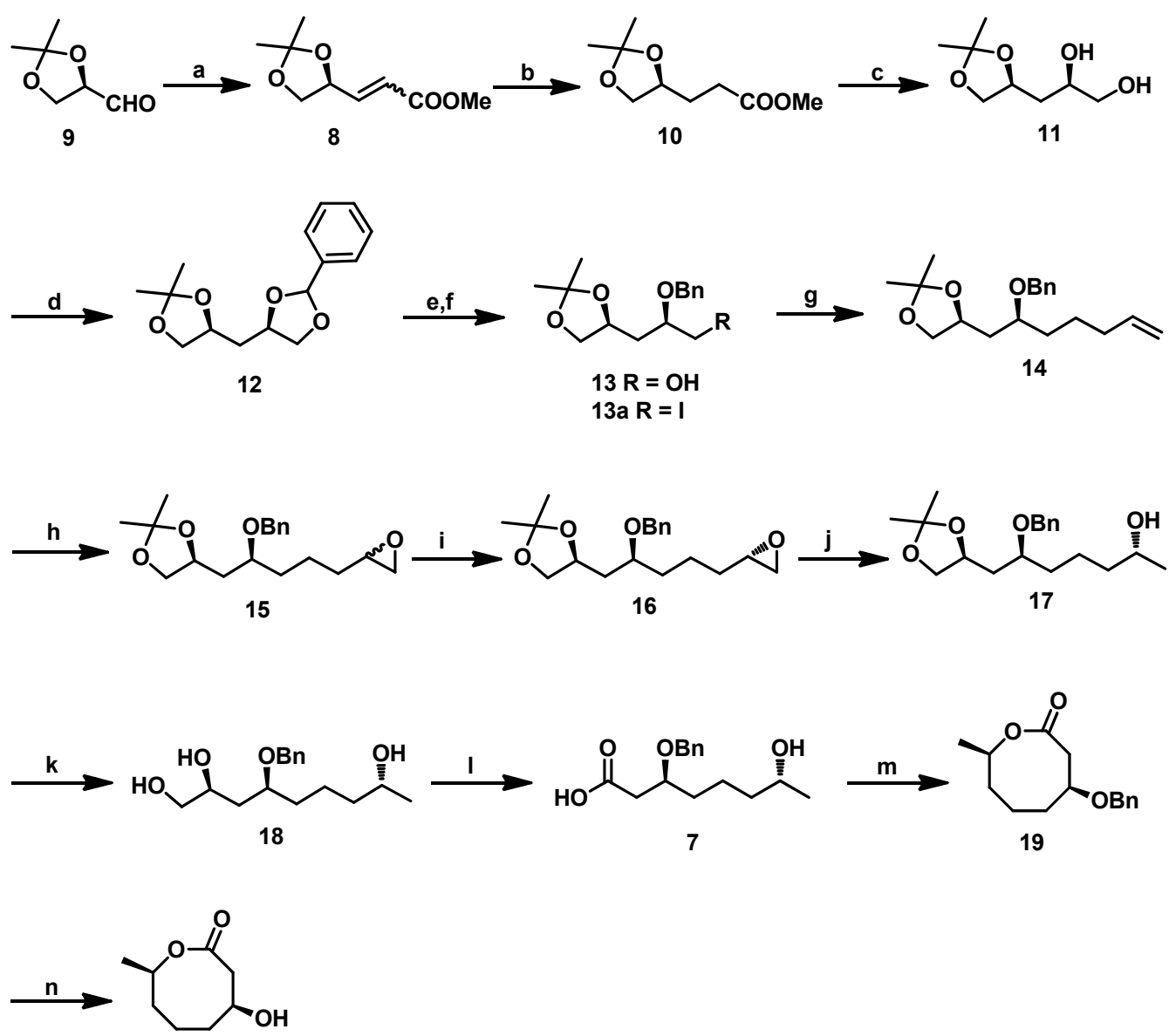

Reagents and conditions: (a) $\mathrm{Ph}_{3} \mathrm{P}=\mathrm{CHCOOMe}$, Benzene, reflux, 2 h, 81\%; (b) $\mathrm{H}_{2}, \mathrm{Pd} / \mathrm{C}, \mathrm{MeOH}, \mathrm{rt}, 3 \mathrm{~h}, 84 \%$; (c) i) $\mathrm{DIBAL}^{-\mathrm{H}}, \mathrm{CH}_{2} \mathrm{Cl}_{2}, 0$ oC to rt, 3 h,; ii) $\mathrm{PhNO}$, Lproline, $\mathrm{CH}_{3} \mathrm{CN},-20^{\circ} \mathrm{C}, 24 \mathrm{~h}$ then $\mathrm{NaBH}_{4}, \mathrm{MeOH}, 0{ }^{\circ} \mathrm{C}$; iii) $10 \% \mathrm{Pd} / \mathrm{C}, \mathrm{H}_{2}, \mathrm{MeOH}, 24 \mathrm{~h}$, overall yield for 3 steps $72 \%$; (d) Benzaldehyde dimethyl acetal, $\mathrm{PTSA}$, $\mathrm{CH}_{2} \mathrm{Cl}{ }_{2}$, $0{ }^{\circ} \mathrm{C}$ to rt, $6 \mathrm{~h}, 83 \%$; (e) DIBAL-H, $\mathrm{CH}_{2} \mathrm{Cl}_{2}, 0{ }^{\circ} \mathrm{C}$ to $\mathrm{rt}, 4 \mathrm{~h}, 79 \%$; (f) $\mathrm{I}_{2}, \mathrm{PPh}_{3}$, imidazole, $0{ }^{\circ} \mathrm{C}$ to $\mathrm{rt}, \mathrm{THF}, 71 \%$; (g) homoallyl bromide, $\mathrm{Mg}$, THF, $0{ }^{\circ} \mathrm{C}, 8 \mathrm{~h}, 83 \%$; (h) $m$ -

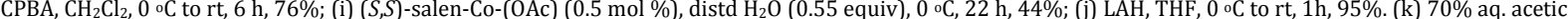
acid, rt, 12 h; (l) i) $\mathrm{NaIO}_{4}$, sat. $\mathrm{NaHCO}_{3}$ soln., $\mathrm{CH}_{2} \mathrm{Cl}_{2}$, rt, 6 h; ii) $\mathrm{NaClO}_{2}, \mathrm{NaH}_{2} \mathrm{PO}_{4}$, 2-methyl-2-butene, $t$-BuOH:water (2:1), 0 ०C to rt, 3 h; (m) 2,4,6-trichlorobenzoyl chloride, $\mathrm{Et}_{3} \mathrm{~N}$, THF, DMAP, toluene, $\mathrm{rt}, 12 \mathrm{~h}$; (n) $\mathrm{TiCl}_{4}, \mathrm{CH}_{2} \mathrm{Cl}_{2}$.

Scheme 1

\subsection{Synthesis}

\subsection{1. (S)-Methyl 3-(2,2-dimethyl-1,3-dioxolan-4-yl)acrylate} (8)

To a stirred solution of compound $9(2.7 \mathrm{~g}, 20.71 \mathrm{mmol})$ in benzene $(50 \mathrm{~mL})$ was treated with (methoxy-carbonyl methylene) triphenyl phosphorane (3.54 g, $10.54 \mathrm{mmol})$ at reflux temperature. After $2 \mathrm{~h}$, solvent was evaporated and purification of the residue by column chromatography (60-120 Silica gel, 15\% EtOAc in petroleum ether) furnished compound 8 (Scheme 1).

(S)-Methyl 3-(2,2-dimethyl-1,3-dioxolan-4-yl)acrylate (8): Color: Yellow liquid. Yield: $81 \% .{ }^{1} \mathrm{H}$ NMR $\left(300 \mathrm{MHz}, \mathrm{CDCl}_{3}, \delta\right.$, ppm): $6.72\left(\mathrm{dd}, 1 \mathrm{H}, J=6.1 \mathrm{~Hz}, 12.2 \mathrm{~Hz},-\mathrm{CH}=\mathrm{CH}_{2}\right), 5.72(\mathrm{~d}, 1 \mathrm{H}, J$ $\left.=12.2 \mathrm{~Hz},-\mathrm{CH}=\mathrm{CH}_{2}\right), 5.34-5.23(\mathrm{~m}, 1 \mathrm{H},-\mathrm{OCH}-), 4.25-4.18(\mathrm{~m}$, $\left.2 \mathrm{H},-\mathrm{OCH}_{2}-\right), 3.68\left(\mathrm{~s}, 3 \mathrm{H}, \mathrm{OCH}_{3}\right), 1.37\left(\mathrm{~s}, 3 \mathrm{H},-\mathrm{CH}_{3}\right), 1.35(\mathrm{~s}, 3 \mathrm{H}$, $\left.\mathrm{CH}_{3}\right)$. ESI-MS $(\mathrm{m} / \mathrm{z}): 209(\mathrm{M}+\mathrm{Na})^{+}$.

\subsection{2. (S)-Methyl 3-(2,2-dimethyl-1,3-dioxolan-4-yl) propanoate $(10)$}

A solution of compound $8(7.0 \mathrm{~g}, 24.47 \mathrm{mmol})$ in methanol $(25.0 \mathrm{~mL})$ was treated with $10 \% \mathrm{Pd}-\mathrm{C}(0.45 \mathrm{~g})$ for $3 \mathrm{~h}$ under hydrogen atmosphere. After completion of reaction, it was filtered, The filtrate was extracted with EtOAc $(2 \times 50 \mathrm{~mL})$, the organic layer was dried $\left(\mathrm{Na}_{2} \mathrm{SO}_{4}\right)$, evaporated and purified by column chromatography (Silica gel, 60-120 mesh, 5\% EtOAc in petroleum ether) to give compound $\mathbf{1 0}$ (Scheme 1).

(S)-Methyl 3-(2,2-dimethyl-1,3-dioxolan-4-yl)propanoate (10): Color: Pale yellow liquid. Yield: $84 \% .{ }^{1} \mathrm{H}$ NMR $(300 \mathrm{MHz}$, $\left.\mathrm{CDCl}_{3}, \delta, \mathrm{ppm}\right): 4.12-4.02\left(\mathrm{~m}, 2 \mathrm{H},-\mathrm{OCH}_{2}-\right), 3.92(\mathrm{~m}, 1 \mathrm{H},-\mathrm{OCH}-)$, $3.64\left(\mathrm{~s}, 3 \mathrm{H}, \mathrm{OCH}_{3}\right), 2.48-2.35\left(\mathrm{~m}, 2 \mathrm{H},-\mathrm{CH}_{2}-\mathrm{C}=0\right), 1.88-1.77(\mathrm{~m}$, $\left.2 \mathrm{H},-\mathrm{CH}_{2}-\right), 1.39\left(\mathrm{~s}, 3 \mathrm{H}, \mathrm{CH}_{3}\right), 1.35\left(\mathrm{~s}, 3 \mathrm{H}, \mathrm{CH}_{3}\right)$. ESI-MS $(\mathrm{m} / \mathrm{z})$ : $211(\mathrm{M}+\mathrm{Na})^{+}$.

\subsection{3. (R)-3-((S)-2,2-Dimethyl-1,3-dioxolan-4-yl)propane- 1,2-diol (11)}

A solution of ester $\mathbf{1 0}(6.9 \mathrm{~g}, 36.72 \mathrm{mmol})$ in dry $\mathrm{CH}_{2} \mathrm{Cl}_{2}(30$ $\mathrm{mL}$ ) was cooled to $-78{ }^{\circ} \mathrm{C}$ and DIBAL-H (18 mL, $25.82 \mathrm{mmol}, 20$ mol\% in toluene) was added. The resultant solution was stirred at the same temperature for $2 \mathrm{~h}$ and quenched with $\mathrm{MeOH}(5$ $\mathrm{mL})$. The reaction mixture was diluted with EtOAc $(20 \mathrm{~mL})$ aq potassium sodium tartrate $(5 \mathrm{~mL})$ and stirred vigorously at room temperature for an additional $1 \mathrm{~h}$. It was filtered through celite, the filtrate was dried $\left(\mathrm{Na}_{2} \mathrm{SO}_{4}\right)$, concentrated to give the crude aldehyde. 
To a stirred solution of aldehyde (5.8 g, $30.85 \mathrm{mmol})$ and nitrosobenzene ( $3.3 \mathrm{~g}, 30.85 \mathrm{mmol})$ in DMSO $(20 \mathrm{~mL})$ was added L-proline $(0.71 \mathrm{~g}, 6.17 \mathrm{mmol}, 20 \mathrm{~mol} \%)$ in one portion at $25{ }^{\circ} \mathrm{C}$. After $24 \mathrm{~h}$, the temperature was lowered to $0{ }^{\circ} \mathrm{C}$, followed by dilution with anhydrous $\mathrm{MeOH}(30 \mathrm{~mL})$ and the careful addition of excess $\mathrm{NaBH}_{4}(2.46 \mathrm{~g}, 61.7 \mathrm{mmol})$. The reaction was quenched after $10 \mathrm{~min}$ by pouring the reaction mixture into a vigorously stirred biphasic solution of $\mathrm{Et}_{2} \mathrm{O}$ and aqueous $\mathrm{HCl}(1 \mathrm{M})$. The organic layer was separated, and the aqueous phase was extracted with EtOAc $(3 \times 30 \mathrm{~mL})$. The combined organic phase was dried over anhydrous $\mathrm{Na}_{2} \mathrm{SO}_{4}$, concentrated, and purified by column chromatography over silica gel using ethyl acetate:petroleum ether $(40: 60, v: v)$ as eluent to give pure aminoxy alcohol as a pure diastereomer. The aminoxy alcohol $(6.7 \mathrm{~g}, 25.09 \mathrm{mmol})$ was dissolved in EtOAc $(30 \mathrm{~mL})$ and to the solution was added $10 \% \mathrm{Pd} / \mathrm{C}(0.35$ g). The reaction mixture was stirred in a hydrogen atmosphere (1 atm, balloon pressure) for $12 \mathrm{~h}$. After completion of the reaction (monitored by TLC), It was filtered through celite pad. The filtrate was dried $\left(\mathrm{Na}_{2} \mathrm{SO}_{4}\right)$ concentrated and residue purified by column chromatography using petroleum ether:ethyl acetate $(3: 2, v: v)$ as eluent to give pure diol 11 (Scheme 1).

(R)-3-((S)-2,2-Dimethyl-1,3-dioxolan-4-yl)propane-1,2-diol (11): Color: Yellow liquid. Yield: 72\%. IR (Neat, $v, \mathrm{~cm}^{-1}$ ): 3412 , 3018, 2938, 1612, 1513, 1248, 1215. ${ }^{1} \mathrm{H}$ NMR (300 MHz, $\mathrm{CDCl}_{3}$, $\delta, \mathrm{ppm}): 4.09-3.92\left(\mathrm{~m}, 4 \mathrm{H}, 2 \mathrm{x}-\mathrm{OCH}_{2}\right), 3.87-3.79(\mathrm{~m}, 2 \mathrm{H}, 2 \mathrm{x}-$ $\mathrm{OCH}$ ), 2.08 (br s, 2H, 2 x -OH) , 1.74-1.59 (m, 2H, $-\mathrm{CH}_{2}$ ), 1.38 (s, $\left.3 \mathrm{H},-\mathrm{CH}_{3}\right), 1.33\left(\mathrm{~s}, 3 \mathrm{H},-\mathrm{CH}_{3}\right) .{ }^{13} \mathrm{C} \mathrm{NMR}\left(75 \mathrm{MHz}, \mathrm{CDCl}_{3}, \delta, \mathrm{ppm}\right):$ 109.1 (1C, -O-C-O-), 74.3 (2C, - O- $\mathrm{CH}-$ ), 72.6 (1C, $\left.-\mathrm{CH}_{2}-\mathrm{OH}\right), 66.8$ $\left(1 \mathrm{C},-0-\mathrm{CH}_{2}-\right), 66.1(1 \mathrm{C},-\mathrm{CH}-\mathrm{OH}), 36.5\left(1 \mathrm{C},-\mathrm{CH}_{2}-\right), 25.7(1 \mathrm{C}$, $\left.\mathrm{CH}_{3}\right)$. ESI-MS $(\mathrm{m} / \mathrm{z}): 199(\mathrm{M}+\mathrm{Na})^{+}$. [ $\left.\alpha\right]_{\mathrm{D}^{2}}{ }^{25}:-66.8\left(\mathrm{c} 0.5, \mathrm{CHCl}_{3}\right)$.

\subsection{4. (4S)-2,2-Dimethyl-4-(((4R)-2-phenyl-1,3-dioxolan-4- yl)methyl)-1,3-dioxolane (12)}

To a stirred solution of compound $\mathbf{1 1}(3.1 \mathrm{~g}, 17.61 \mathrm{mmol})$ in dry $\mathrm{CH}_{2} \mathrm{Cl}_{2}(20 \mathrm{~mL})$, benzaldehyde dimethylacetal $(3.2 \mathrm{~mL}$, $21.13 \mathrm{mmol}$ ) followed by $p$-toluedine sulphonic acid (PTSA, catalytic) were added at $0{ }^{\circ} \mathrm{C}$. After stirring of $6 \mathrm{~h}$ the reaction mixture was treated with aq. $\mathrm{NaHCO}_{3}(10 \mathrm{~mL})$ and extracted with $\mathrm{CH}_{2} \mathrm{Cl}_{2}(3 \times 30 \mathrm{~mL})$ and both the organic layers were dried over anhydrous $\mathrm{Na}_{2} \mathrm{SO} 4$ and concentrated. Crude product was purified by column chromatography (60-120 Silica gel, 10\% EtOAc in petroleum ether) to afford compound 12 (Scheme 1).

(4S)-2,2-Dimethyl-4-(((4R)-2-phenyl-1,3-dioxolan-4-yl) methyl)-1,3-dioxolane (12): Color: Pale yellow syrup. Yield: $83 \%$. IR (Neat, $\left.v, \mathrm{~cm}^{-1}\right): 3010,2900,1250,852 .{ }^{1} \mathrm{H}$ NMR $(300$ $\left.\mathrm{MHz}, \mathrm{CDCl}_{3}, \delta, \mathrm{ppm}\right): 7.35-7.29\left(\mathrm{~m}, 5 \mathrm{H},-\mathrm{C}_{6} \mathrm{H}_{5}\right), 5.77(\mathrm{~s}, 1 \mathrm{H},-$ OCHPh), 4.07-3.88 (m, 4H, 2 x -OCH2-), 3.99-3.89 (m, 2H, -OCH), 1.78-1.54 (m, 2H, $\left.-\mathrm{CH}_{2}-\right), 1.36\left(\mathrm{~s}, 3 \mathrm{H},-\mathrm{CH}_{3}\right), 1.31\left(\mathrm{~s}, 3 \mathrm{H},-\mathrm{CH}_{3}\right)$. ${ }^{13} \mathrm{C}$ NMR $\left(75 \mathrm{MHz}, \mathrm{CDCl}_{3}, \delta, \mathrm{ppm}\right): 140.6$ (1C, $\mathrm{Ar}-\mathrm{C}$ ), 129.8 (2C, $\operatorname{Ar}-C), 128.6$ (1C, Ar-C), 126.3 (2C, Ar-C), 108.3 (1C, -O-C-O-), 104.3 (1C, -O-CH-O-), 78.1 (1C, -O-CH-), 74.2 (1C, - O-CH-), 72.2 $\left(1 \mathrm{C},-\mathrm{O}-\mathrm{CH}_{2}-\right), 66.7\left(1 \mathrm{C},-\mathrm{O}-\mathrm{CH}_{2}-\right), 36.4\left(1 \mathrm{C},-\mathrm{CH}_{2}-\right), 25.8(2 \mathrm{C}$, $\left.\mathrm{CH}_{3}\right)$. ESI-MS $(\mathrm{m} / \mathrm{z}): 287(\mathrm{M}+\mathrm{Na})^{+}$.

\subsection{5. (R)-2-(Benzyloxy)-3-((S)-2,2-dimethyl-1,3-dioxolan-4- yl)propan-1-ol (13)}

To a stirred solution of compound $12(3.6 \mathrm{~g}, 13.63 \mathrm{mmol})$ in dry $\mathrm{CH}_{2} \mathrm{Cl}_{2}(20 \mathrm{~mL})$ DIBAL-H $(11.6 \mathrm{~mL}, 16.36 \mathrm{mmol}, 2 \mathrm{M}$ solution in toluene) was added at $0{ }^{\circ} \mathrm{C}$. After $4 \mathrm{~h}$ the reaction mixture was quenched with $\mathrm{MeOH}$ and aq. sodium potassium tartrate $(5 \mathrm{~mL})$, filtered through celite. The residue was washed with $\mathrm{CH}_{2} \mathrm{Cl}_{2}(2 \times 50 \mathrm{~mL})$ and both the organic layers were dried over anhydrous $\mathrm{Na}_{2} \mathrm{SO} 4$ and concentrated. Crude product was purified by column chromatography (60-120 Silica gel, 30\% EtOAc in petroleum ether) to afford compound $\mathbf{1 3}$ (Scheme 1).

(R)-2-(Benzyloxy)-3-((S)-2,2-dimethyl-1,3-dioxolan-4-yl) propan-1-ol (13): Color: Colorless syrup. Yield: 79\%. IR (Neat, $v$, $\left.\mathrm{cm}^{-1}\right): 3450,3010,2900,1250,1050,850 .{ }^{1} \mathrm{H}$ NMR (300 MHz, $\left.\mathrm{CDCl}_{3}, \delta, \mathrm{ppm}\right): 7.32-7.27\left(\mathrm{~m}, 5 \mathrm{H},-\mathrm{C}_{6} \mathrm{H}_{5}\right), 4.58\left(\mathrm{~s}, 2 \mathrm{H},-\mathrm{OCH}_{2} \mathrm{Ph}\right)$, 4.12-4.08 (m, 4H, 2 x- $\left.-\mathrm{OCH}_{2}\right), 3.76-3.68(\mathrm{~m}, 1 \mathrm{H},-\mathrm{OCH}), 3.49-3.41$ (m, $1 \mathrm{H},-\mathrm{OCH}), 1.78-1.58\left(\mathrm{~m}, 3 \mathrm{H},-\mathrm{CH}_{2}, \mathrm{OH}\right), 1.37\left(\mathrm{~s}, 3 \mathrm{H},-\mathrm{CH}_{3}\right)$, $1.34\left(\mathrm{~s}, 3 \mathrm{H},-\mathrm{CH}_{3}\right) .{ }^{13} \mathrm{C} \mathrm{NMR}\left(75 \mathrm{MHz}, \mathrm{CDCl}_{3}, \delta, \mathrm{ppm}\right): 138.8(1 \mathrm{C}$ Ar-C), 127.9 (2C, Ar-C), 127.6 (3C, Ar-C), 109.3 (1C, -O-C-O-), 76.5 (1C, - $\mathrm{O}-\mathrm{CH}-\mathrm{O}-)$, 74.3 (1C, -O-CH-), 72.6 (1C, -O- $\mathrm{CH}_{2}-$ ), 66.7 (1C, $\left.-\mathrm{CH}_{2}-\mathrm{OH}\right), 66.5\left(1 \mathrm{C},-\mathrm{CH}_{2}-\mathrm{OH}\right), 36.8\left(1 \mathrm{C},-\mathrm{CH}_{2}-\right), 25.1(2 \mathrm{C}$, $\left.\mathrm{CH}_{3}\right)$. ESI-MS $(\mathrm{m} / \mathrm{z}): 289(\mathrm{M}+\mathrm{Na})^{+} .[\alpha]_{\mathrm{D}}{ }^{25}:-70.92\left(c 0.27, \mathrm{CHCl}_{3}\right)$.

\subsection{6. (S)-4-((S)-2-(Benzyloxy)hept-6-enyl)-2,2-dimethyl-1,3- dioxolane (14)}

A solution of alcohol 13 (2.76 g, $10.37 \mathrm{mmol})$ in dry THF $(20 \mathrm{~mL})$, imidazole $(1.1 \mathrm{~g}, 15.55 \mathrm{mmol}), \mathrm{Ph}_{3} \mathrm{P}$ (3.25 g, 12.44 mmol) and iodine (1.31 g, $10.37 \mathrm{mmol})$ were added at $0{ }^{\circ} \mathrm{C}$ and allowed to stir for $1 \mathrm{~h}$. After that it was neutralized with aq. $\mathrm{NaHCO}_{3}(10 \mathrm{~mL})$ solution and extracted with $10 \%$ EtOAc in hexane $(2 \times 30 \mathrm{~mL})$. The combined organic layers were dried over anhydrous $\mathrm{Na}_{2} \mathrm{SO}_{4}$ and concentrated under vacuo. The residue was purified by flash column chromatography (Silica gel, 60-120 mesh, 5\% EtOAc in petroleum ether) to afford compound 13a $(2.7 \mathrm{~g}, 71 \%)$ as a yellow colored liquid.

The iodo derivative 13a $(2.7 \mathrm{~g}, 7.18 \mathrm{mmol})$ was immediately treated with a solution of CuI $(1.36 \mathrm{~g}, 7.18 \mathrm{mmol})$ and homoallylmagnesium bromide (prepared from homoallyl bromide $(12.6 \mathrm{~mL}, 97.5 \mathrm{mmol})$ and $\mathrm{Mg}(6.08 \mathrm{~g}, 264.0 \mathrm{mmol})$ in dry THF) at $-40{ }^{\circ} \mathrm{C}$. After stirring at $-40^{\circ} \mathrm{C}$ for $8 \mathrm{~h}$, it was treated with aq. $\mathrm{NH}_{4} \mathrm{Cl}(20 \mathrm{~mL})$. The residue was extracted with EtOAc $(2 \times 30 \mathrm{~mL})$ and the organic layers were dried over anhydrous $\mathrm{Na}_{2} \mathrm{SO} 4$ and concentrated. Crude product was purified by column chromatography (Silica gel, 60-120 mesh, 5\% EtOAc in petroleum ether) to afford compound $\mathbf{1 4}$ (Scheme 1).

(S)-4-((S)-2-(Benzyloxy)hept-6-enyl)-2,2-dimethyl-1,3-dioxo lane (14): Color: Colorless liquid. Yield: $83 \%$. IR (Neat, $v, \mathrm{~cm}^{-1}$ ): $3435,3070,2932,2829,1635,1466,1134,1015 .{ }^{1} \mathrm{H}$ NMR $(300$ $\left.\mathrm{MHz}, \mathrm{CDCl}_{3}, \delta, \mathrm{ppm}\right): 7.32-7.26\left(\mathrm{~m}, 5 \mathrm{H},-\mathrm{C}_{6} \mathrm{H}_{5}\right), 5.79-5.66(\mathrm{~m}, 1 \mathrm{H}$, $\left.-\mathrm{CH}=\mathrm{CH}_{2}\right), 4.98-4.89\left(\mathrm{~m}, 2 \mathrm{H},-\mathrm{CH}=\mathrm{CH}_{2}\right), 4.56(\mathrm{~d}, 1 \mathrm{H}, J=11.3 \mathrm{~Hz},-$ $\left.\mathrm{OCH}_{2} \mathrm{Ph}\right), 4.44\left(\mathrm{~d}, 1 \mathrm{H}, J=11.4 \mathrm{~Hz},-\mathrm{OCH}_{2} \mathrm{Ph}\right), 4.07-3.99(\mathrm{~m}, 2 \mathrm{H}$, $\left.\mathrm{OCH}_{2}\right), 3.77-3.66(\mathrm{~m}, 1 \mathrm{H},-\mathrm{OCH}), 3.52-3.44(\mathrm{~m}, 1 \mathrm{H},-\mathrm{OCH}), 2.16-$ $2.09\left(\mathrm{~m}, 2 \mathrm{H},-\mathrm{CH}_{2}\right), 1.61-1.31\left(\mathrm{~m}, 6 \mathrm{H}, 3 \mathrm{x}-\mathrm{CH}_{2}\right), 1.36(\mathrm{~s}, 3 \mathrm{H},-$ $\left.\mathrm{CH}_{3}\right), 1.32$ (s, 3H, $\left.\left.-\mathrm{CH}_{3}\right) .{ }^{13} \mathrm{C} \mathrm{NMR} \mathrm{(75} \mathrm{MHz,} \mathrm{CDCl}_{3}, \delta, \mathrm{ppm}\right): 138.8$ (1C, $\mathrm{Ar}-\mathrm{C}), 133.7\left(1 \mathrm{C},-\mathrm{CH}=\mathrm{CH}_{2}\right), 128.7$ (2C, $\left.\mathrm{Ar}-\mathrm{C}\right), 128.3$ (1C, $\mathrm{Ar}-$ C), $128.1\left(2 \mathrm{C}, \mathrm{Ar}-\mathrm{C}\right.$ ), $114.8\left(1 \mathrm{C},=\mathrm{CH}_{2}\right), 108.5$ (1C, $\left.-0-C-0-\right), 77.3$ (1C, $-\mathrm{O}-\mathrm{CH}-$ ), 74.2 (1C, $-\mathrm{O}-\mathrm{CH}-), 72.1\left(1 \mathrm{C},-0-\mathrm{CH}_{2}-\right), 66.5$ (1C, - $0-$ $\mathrm{CH}_{2}-$ ), 39.8 (1C, - $\mathrm{CH}_{2}-$ ), 34.2 (1C, $-\mathrm{CH}_{2}-$ ), 33.8 (1C, $-\mathrm{CH}_{2}-$ ), 32.7 $\left(1 \mathrm{C},-\mathrm{CH}_{2}-\right), 25.2\left(2 \mathrm{C},-\mathrm{CH}_{3}\right)$. ESI-MS $(\mathrm{m} / \mathrm{z}):(\mathrm{M}+\mathrm{Na})+327 .[\alpha]_{\mathrm{D}^{25}}$ : $+39.71\left(c 1.12, \mathrm{CHCl}_{3}\right)$.

\subsection{7. (S)-4-((S)-2-(Benzyloxy)-5-(oxiran-2-yl)pentyl)-2,2- dimethyl-1,3-dioxolane (15)}

To a stirred solution of olefin $\mathbf{1 4}(2.5 \mathrm{~g}, 8.22 \mathrm{mmol})$ in $\mathrm{CHCl}_{3}$ (25 mL), $m$-chloroperbenzoic acid (m-CPBA) (2.12 g, 12.33 $\mathrm{mmol}$ ) was added and allowed to stir for $24 \mathrm{~h}$ at room temperature. After that the reaction mixture was filtered and the filtrate was washed with saturated $\mathrm{NaHCO}_{3}$ solution $(30$ $\mathrm{mL}$ ), dried over $\mathrm{Na}_{2} \mathrm{SO}_{4}$ and concentrated. Crude product was purified by column chromatography $(60-120$ Silica gel, $10 \%$ EtOAc in petroleum ether) to furnish compound 15 (Scheme 1).

(S)-4-((S)-2-(Benzyloxy)-5-(oxiran-2-yl)pentyl)-2,2-dimethyl1,3-dioxolane (15): Color: Colorless liquid. Yield: 76\%. IR (Neat, $\left.v, \mathrm{~cm}^{-1}\right): 3050,2932,1620,1452,1034,950,860 .{ }^{1} \mathrm{H}$ NMR $(300$ $\left.\mathrm{MHz}, \mathrm{CDCl}_{3}, \delta, \mathrm{ppm}\right): 7.35-7.24\left(\mathrm{~m}, 5 \mathrm{H},-\mathrm{C}_{6} \mathrm{H}_{5}\right), 4.46(\mathrm{dd}, 2 \mathrm{H}, J=$ $\left.11.6 \mathrm{~Hz}, 5.8 \mathrm{~Hz}-\mathrm{OC}_{2} \mathrm{Ph}\right), 4.06-3.95\left(\mathrm{~m}, 2 \mathrm{H},-\mathrm{OCH}_{2}\right), 3.79-3.70$ (m, 1H, -OCH), 3.49-3.41 (m, 1H, -OCH), 2.89-2.81 (m, 1H, epoxy $\mathrm{CH}), 2.68-2.62(\mathrm{~m}, 1 \mathrm{H}$, epoxy $\mathrm{CH}), 2.49(\mathrm{~m}, 1 \mathrm{H}$, epoxy $\mathrm{CH}), 1.63$ $\left(\mathrm{m}, 2 \mathrm{H}, \mathrm{CH}_{2}\right), 1.37\left(\mathrm{~m}, 2 \mathrm{H}, \mathrm{CH}_{2}\right), 1.36\left(\mathrm{~s}, 3 \mathrm{H},-\mathrm{CH}_{3}\right), 1.32(\mathrm{~s}, 3 \mathrm{H},-$ $\left.\mathrm{CH}_{3}\right), 1.24\left(\mathrm{~m}, 2 \mathrm{H}, \mathrm{CH}_{2}\right), 1.22-1.11\left(\mathrm{~m}, 2 \mathrm{H},-\mathrm{CH}_{2}\right)$. ESI-MS $(\mathrm{m} / \mathrm{z})$ : $343(\mathrm{M}+\mathrm{Na})^{+}$. 
2.2.8. (S)-4-((S)-2-(Benzyloxy)-5-((S)-oxiran-2-yl)pentyl)-2,2dimethyl-1,3-dioxolane (16)

A mixture of $(S, S)-N, N$-bis(3,5-di-tert-butylsalicylidine)-1,2cyclohexanediamino Co(III) chloride complex $(0.18 \mathrm{~g}, 0.29$ $\mathrm{mmol})$ and $\mathrm{AcOH}(0.035 \mathrm{~g}, 0.59 \mathrm{mmol})$ in toluene $(1 \mathrm{~mL})$ was stirred in open air for $1 \mathrm{~h}$ at room temperature. Then the solvent was removed by rotary evaporator, and the resulting brown residue was dried under vacuum. To this activated catalyst, epoxide 15 (1.95 g, $5.93 \mathrm{mmol})$ was added in one portion and it was cooled in an ice-water bath. Then $\mathrm{H}_{2} \mathrm{O}(0.058$ $\mathrm{g}, 3.26 \mathrm{mmol}$ ) was slowly added to the reaction mixture. Stirring was continued for $1 \mathrm{~h}$ in an ice-water bath. After that, ice-water bath was removed and the reaction mixture was allowed to stir at room temperature for $12 \mathrm{~h}$. The crude reaction mixture was adsorbed on silica gel and purified by column chromatography (60-120 Silica gel, 40\% EtOAc in petroleum ether) to give compound $\mathbf{1 6}$ (Scheme 1).

(S)-4-((S)-2-(Benzyloxy)-5-((S)-oxiran-2-yl)pentyl)-2,2dimethyl-1,3-dioxolane (16): Color: Yellow liquid. Yield: 44\%. ${ }^{1} \mathrm{H}$ NMR $\left(300 \mathrm{MHz}, \mathrm{CDCl}_{3}, \delta, \mathrm{ppm}\right): 7.35-7.23\left(\mathrm{~m}, 5 \mathrm{H},-\mathrm{C}_{6} \mathrm{H}_{5}\right)$, $4.49\left(\mathrm{~d}, 1 \mathrm{H}, J=11.5 \mathrm{~Hz},-\mathrm{OCH}_{2} \mathrm{Ph}\right), 4.44(\mathrm{~d}, 1 \mathrm{H}, J=11.5 \mathrm{~Hz}$, $\left.\mathrm{OCH}_{2} \mathrm{Ph}\right)$, 4.06-3.95 (m, $\left.2 \mathrm{H},-\mathrm{OCH}_{2}\right), 3.79-3.71(\mathrm{~m}, 1 \mathrm{H},-\mathrm{OCH})$, $3.49-3.38(\mathrm{~m}, 1 \mathrm{H},-\mathrm{OCH}), 2.89$ (dt, $1 \mathrm{H}, J=4.8,7.2 \mathrm{~Hz}$, epoxy $\mathrm{CH}$ ), 2.68 (dd, $1 \mathrm{H}, J=4.8,5.2 \mathrm{~Hz}$, epoxy $\mathrm{CH}$ ), 2.49 (dd, $1 \mathrm{H}, J=2.8,5.2$ $\mathrm{Hz}$, epoxy $\mathrm{CH}), 1.61-1.38\left(\mathrm{~m}, 6 \mathrm{H}, 3 \mathrm{x}-\mathrm{CH}_{2}\right), 1.36\left(\mathrm{~s}, 3 \mathrm{H},-\mathrm{CH}_{3}\right)$, $1.32\left(\mathrm{~s}, 3 \mathrm{H},-\mathrm{CH}_{3}\right), 1.22-1.17\left(\mathrm{~m}, 2 \mathrm{H},-\mathrm{CH}_{2}\right)$. ESI-MS $(\mathrm{m} / \mathrm{z}): 343$ $(\mathrm{M}+\mathrm{Na})^{+}$.

\subsection{9. (2R,6S)-6-(Benzyloxy)-7-((S)-2,2-dimethyl-1,3- dioxolan-4-yl)heptan-2-ol (17)}

To a cooled $\left(0{ }^{\circ} \mathrm{C}\right)$ and stirred suspension of lithium aluminium hydride (LAH) $(0.052 \mathrm{~g}, 1.380 \mathrm{mmol})$ in dry THF (4 $\mathrm{mL})$, at $0{ }^{\circ} \mathrm{C}$ was added compound $16(0.290 \mathrm{~g}, 0.690 \mathrm{mmol})$ in THF $(2 \mathrm{~mL})$ and allowed to stir at room temperature for $1 \mathrm{~h}$. The reaction mixture was quenched by careful addition of aq. $\mathrm{Na}_{2} \mathrm{SO}_{4}$ solution at $0{ }^{\circ} \mathrm{C}$, filtered through a pad of celite and filtrate concentrated under reduced pressure. The crude residue was purified by column chromatography (60-120 silica gel, 1:3 EtOAc: $n$-Hexane) to give compound 17 (Scheme 1).

(2R,6S)-6-(Benzyloxy)-7-((S)-2,2-dimethyl-1,3-dioxolan-4-yl) heptan-2-ol (17): Color: Colorless syrup. Yield: 95\%. IR (Neat, $v$, $\left.\mathrm{cm}^{-1}\right)$ : 3446, 2933, 1611, 1523, 1455, 1374, 1093, 928. ${ }^{1} \mathrm{H}$ NMR (300 MHz, $\left.\mathrm{CDCl}_{3}, \delta, \mathrm{ppm}\right): 7.36-7.24\left(\mathrm{~m}, 5 \mathrm{H},-\mathrm{C}_{6} \mathrm{H}_{5}\right), 4.51(\mathrm{~d}, 1 \mathrm{H}$, $\left.J=11.8 \mathrm{~Hz},-\mathrm{OCH}_{2} \mathrm{Ph}\right), 4.46\left(\mathrm{~d}, 1 \mathrm{H}, J=11.8 \mathrm{~Hz},-\mathrm{OCH}_{2} \mathrm{Ph}\right), 4.09-$ $3.98\left(\mathrm{~m}, 2 \mathrm{H},-\mathrm{OCH}_{2}\right), 3.76-3.67(\mathrm{~m}, 2 \mathrm{H}, 2 \mathrm{x}-\mathrm{OCH}), 3.51-3.44(\mathrm{~m}$, $1 \mathrm{H},-\mathrm{OCH}), 1.74-1.58\left(\mathrm{~m}, 7 \mathrm{H}, 3 \mathrm{x}-\mathrm{CH}_{2}, \mathrm{OH}\right), 1.36\left(\mathrm{~s}, 3 \mathrm{H},-\mathrm{CH}_{3}\right)$, $1.34\left(\mathrm{~s}, 3 \mathrm{H},-\mathrm{CH}_{3}\right), 1.22-1.16\left(\mathrm{~m}, 2 \mathrm{H},-\mathrm{CH}_{2}\right), 1.14(\mathrm{~d}, 3 \mathrm{H}, J=6.3$ $\left.\mathrm{Hz},-\mathrm{CH}_{3}\right) .{ }^{13} \mathrm{C}$ NMR $\left(75 \mathrm{MHz}, \mathrm{CDCl}_{3}, \delta, \mathrm{ppm}\right): 139.1$ (1C, $\mathrm{Ar}-\mathrm{C}$ ), 128.7 (2C, $\mathrm{Ar}-\mathrm{C}$ ), 128.3 (1C, $\mathrm{Ar}-\mathrm{C}), 128.1$ (2C, $\mathrm{Ar}-\mathrm{C}$ ), 108.3 (1C, O-C-O-), $78.4(1 \mathrm{C},-\mathrm{O}-\mathrm{CH}-), 74.6(1 \mathrm{C},-\mathrm{O}-\mathrm{CH}-), 72.4\left(1 \mathrm{C},-\mathrm{O}-\mathrm{CH}_{2}-\right)$ $66.4\left(1 \mathrm{C},-\mathrm{O}-\mathrm{CH}_{2}-\right), 66.0$ (1C, $\left.-\mathrm{CH}-\mathrm{OH}\right), 39.7\left(1 \mathrm{C},-\mathrm{CH}_{2}-\right), 37.8$ (1C, $-\mathrm{CH}_{2}-$ ), $33.4\left(1 \mathrm{C},-\mathrm{CH}_{2}-\right), 25.6\left(2 \mathrm{C},-\mathrm{CH}_{3}\right), 22.1\left(1 \mathrm{C},-\mathrm{CH}_{3}\right), 20.3$ (1C, $-\mathrm{CH}_{2}-$ ). ESI-MS $(\mathrm{m} / \mathrm{z}): 345(\mathrm{M}+\mathrm{Na})^{+} .[\alpha]_{\mathrm{D}}{ }^{25}:+28.3(c \mathrm{c} 0.49$, $\left.\mathrm{CHCl}_{3}\right)$.

\subsubsection{0. (2S,4S,8R)-4-(Benzyloxy)nonane-1,2,8-triol (18)}

A solution of compound 17 (0.350 g, $1.143 \mathrm{mmol})$ in aq. $60 \%$ acetic acid $(10 \mathrm{~mL})$ was stirred at room temperature for $12 \mathrm{~h}$. After completion of reaction, it was quenched with $\mathrm{NaHCO}_{3}$ and adjusted to $\mathrm{pH}=2-3$. The reaction mixture was extracted with ethyl acetate $(3 \mathrm{x} \times 10 \mathrm{~mL})$ and dried $\left(\mathrm{Na}_{2} \mathrm{SO}_{4}\right)$. Evaporation of solvent under reduced pressure and purification of the residue by column chromatography (Silica gel, 60-120 mesh, 40\% EtOAc in petroleum ether) to afford compound 18 (Scheme 1).

(2S,4S,8R)-4-(Benzyloxy)nonane-1,2,8-triol (18): Color: Colorless syrup. Yield: $88 \%$. IR (Neat, $v, \mathrm{~cm}^{-1}$ ): 3449, 3320, $3049,2933,1612,1512,1451,1398,1075 .{ }^{1} \mathrm{H}$ NMR $(300 \mathrm{MHz}$, $\left.\mathrm{CDCl}_{3}, \delta, \mathrm{ppm}\right): 7.32-7.26\left(\mathrm{~m}, 5 \mathrm{H},-\mathrm{C}_{6} H_{5}\right), 4.47$ (s, $\left.2 \mathrm{H},-\mathrm{OCH}_{2} \mathrm{Ph}\right)$,
3.92-3.84 (m, 2H, $\left.-\mathrm{OCH}_{2}\right), 3.79-3.68(\mathrm{~m}, 2 \mathrm{H}, 2 \mathrm{x}-\mathrm{OCH}), 3.49-3.38$ (m, 1H, -OCH), 2.95 (br.s, 2H, 2 x -OH), 1.68-1.49 (m, 7H, $3 \mathrm{x}-$ $\left.\mathrm{CH}_{2}, \mathrm{OH}\right), 1.19-1.13\left(\mathrm{~m}, 2 \mathrm{H},-\mathrm{CH}_{2}\right), 1.12\left(\mathrm{~d}, 3 \mathrm{H}, \mathrm{J}=6.1 \mathrm{~Hz},-\mathrm{CH}_{3}\right)$. ${ }^{13} \mathrm{C}$ NMR $\left(75 \mathrm{MHz}, \mathrm{CDCl}_{3}, \delta, \mathrm{ppm}\right.$ ): 139.3 (1C, $\mathrm{Ar}-\mathrm{C}$ ), 127.9 (2C, $\mathrm{Ar}-\mathrm{C}), 128.4$ (1C, $\mathrm{Ar}-\mathrm{C}), 128.1$ (2C, Ar-C), 76.8 (1C, -O- $\mathrm{CH}-), 72.2$ (1C, $\left.-0-\mathrm{CH}_{2}-\right), 69.8$ (1C, $\left.-\mathrm{CH}-\mathrm{OH}\right), 68.3\left(1 \mathrm{C},-\mathrm{CH}_{2}-\mathrm{OH}\right), 67.2(1 \mathrm{C}$, $\mathrm{CH}_{2}-\mathrm{OH}, 40.3\left(1 \mathrm{C},-\mathrm{CH}_{2}-\right), 38.3\left(1 \mathrm{C},-\mathrm{CH}_{2}-\right), 33.4\left(1 \mathrm{C},-\mathrm{CH}_{2}-\right)$, 23.5(1C, $\left.-\mathrm{CH}_{3}\right), 22.4\left(1 \mathrm{C},-\mathrm{CH}_{2}-\right)$. ESI-MS $(\mathrm{m} / \mathrm{z}): 305(\mathrm{M}+\mathrm{Na})^{+}$. $[\alpha]_{\mathrm{D}}^{25}:+41.3\left(\right.$ c $\left.0.65, \mathrm{CHCl}_{3}\right)$.

\subsubsection{1. (3S,7R)-3-(Benzyloxy)-7-hydroxyoctanoic acid (7)}

To a cooled $\left(0{ }^{\circ} \mathrm{C}\right)$ solution of compound $\mathbf{1 8}(10.0 \mathrm{~g}, 37.59$ mmol) in $\mathrm{CH}_{2} \mathrm{Cl}_{2}(100 \mathrm{~mL}), \mathrm{NaIO}_{4}(12.06 \mathrm{~g}, 56.39 \mathrm{mmol})$ followed by sat. $\mathrm{NaHCO}_{3}(4 \mathrm{~mL})$ were added and stirred it at room temperature for $5 \mathrm{~h}$. After completion of reaction, it was dried over $\mathrm{Na}_{2} \mathrm{SO}_{4}$, washed with $\mathrm{CH}_{2} \mathrm{Cl}_{2}(2 \times 50 \mathrm{~mL})$ filtered and evaporated under reduced pressure to give aldehyde quantitatively.

To a cooled $\left(0{ }^{\circ} \mathrm{C}\right)$ solution of the above obtained aldehyde in $t$-butanol ( $4 \mathrm{~mL}), 2$-methyl-2-butene $(2 \mathrm{~mL})$ was added, followed by a solution of $\mathrm{NaClO}_{2}(0.26 \mathrm{~g}, 2.95 \mathrm{mmol})$ and $\mathrm{NaH}_{2} \mathrm{PO}_{4}(0.46 \mathrm{~g}, 2.95 \mathrm{mmol})$ in water $(2 \mathrm{~mL})$ and stirred at room temperature for $3 \mathrm{~h}$. $t$-Butanol was evaporated and extracted with ethyl acetate $(2 \times 10 \mathrm{~mL})$. The organic layers were washed with water $(2 \times 5 \mathrm{~mL})$, brine $(10 \mathrm{~mL})$, dried $\left(\mathrm{Na}_{2} \mathrm{SO}_{4}\right)$, evaporated and purified the residue by column chromatography (60-120 Silica gel, 40\% EtOAc in petroleum ether) to furnish compound 7 (Scheme 1).

(3S,7R)-3-(Benzyloxy)-7-hydroxyoctanoic acid (7): Color: Colorless syrup. Yield: 78\%. IR (Neat, v, $\mathrm{cm}^{-1}$ ): 3402, 3048, 2984, 1710, 1436, 1258, 1092, 777. ${ }^{1} \mathrm{H}$ NMR $\left(300 \mathrm{MHz}, \mathrm{CDCl}_{3}, \delta\right.$, ppm): 10.52 (brs, $1 \mathrm{H},-\mathrm{COOH}), 7.39-7.21\left(\mathrm{~m}, 5 \mathrm{H},-\mathrm{C}_{6} \mathrm{H}_{5}\right), 4.58$ (d, $\left.1 \mathrm{H}, J=11.6 \mathrm{~Hz},-\mathrm{OCH}_{2} \mathrm{Ph}\right), 4.51\left(\mathrm{~d}, 1 \mathrm{H}, J=11.6 \mathrm{~Hz},-\mathrm{OCH}_{2} \mathrm{Ph}\right)$, 3.94-3.85 (m, 2H, CH), 2.67-2.49 (m, 2H, - $\left.\mathrm{CH}_{2}\right), 1.73-1.34$ (m, $\left.7 \mathrm{H}, 3 \times-\mathrm{CH}_{2}+\mathrm{OH}\right), 1.16\left(\mathrm{~d}, 3 \mathrm{H}, J=6.3 \mathrm{~Hz},-\mathrm{CH}_{3}\right) .{ }^{13} \mathrm{C}$ NMR $(75$ $\left.\mathrm{MHz}, \mathrm{CDCl}_{3}, \delta, \mathrm{ppm}\right): 176.8$ (1C, $\left.-\mathrm{COOH}\right), 138.1$ (1C, $\left.\mathrm{Ar}-\mathrm{C}\right), 128.4$ (2C, $\mathrm{Ar}-\mathrm{C}), 128.6(1 \mathrm{C}, \mathrm{Ar}-\mathrm{C}), 127.9(2 \mathrm{C}, \mathrm{Ar}-\mathrm{C}), 75.6(1 \mathrm{C},-\mathrm{CH}-\mathrm{OH})$ $71.5\left(1 \mathrm{C},-\mathrm{CH}_{2}-\mathrm{OH}\right), 67.8(1 \mathrm{C},-\mathrm{CH}-\mathrm{OH}), 39.5\left(1 \mathrm{C},-\mathrm{CH}_{2}-\right), 34.1$ (1C, $\left.-\mathrm{CH}_{2}-\right), 23.5\left(1 \mathrm{C},-\mathrm{CH}_{3}\right), 21.2\left(1 \mathrm{C},-\mathrm{CH}_{2}-\right)$. ESI-MS (m/z): 289 $(\mathrm{M}+\mathrm{Na})^{+} \cdot[\alpha]_{\mathrm{D}}{ }^{25}:-8.1\left(c 0.8, \mathrm{CHCl}_{3}\right)$.

\subsubsection{2. (4S,8R)-4-(Benzyloxy)-8-methyloxocan-2-one (19)}

A solution of hydroxy acid $7(0.19 \mathrm{~g}, 0.64 \mathrm{mmol})$ in dry THF (1 $\mathrm{mL}$ ) was cooled to $0{ }^{\circ} \mathrm{C}$ under $\mathrm{N}_{2}$ atmosphere. Then, triethylamine $(0.3 \mathrm{~mL}, 1.92 \mathrm{mmol})$ and 2,4,6-trichlorobenzoyl chloride $(0.16 \mathrm{~mL}, 0.96 \mathrm{mmol})$ were added dropwise and allowed to stirr at room temperature for $2 \mathrm{~h}$. The mixture was then diluted with toluene $(10 \mathrm{~mL})$ and stirred at room temperature for further $1.5 \mathrm{~h}$. Subsequently, this mixture was added dropwise over $8 \mathrm{~h}$ to a stirred solution of DMAP $(0.15 \mathrm{~g}$, $1.28 \mathrm{mmol})$ in toluene $(350 \mathrm{~mL})$ at $90{ }^{\circ} \mathrm{C}$. After the complete addition, the reaction mixture was further stirred at $100{ }^{\circ} \mathrm{C}$ for $1 \mathrm{~h}$. It was cooled to room temperature and concentrated under reduced pressure. Purification of the residue by column chromatography (60-120 Silica gel, 6\% EtOAc in petroleum ether) to give compound 19 (Scheme 1).

(4S,8R)-4-(Benzyloxy)-8-methyloxocan-2-one (19): Color: yellow syrup. Yield: 64\%. IR (Neat, $\left.v, \mathrm{~cm}^{-1}\right): 2924,2854,1719$, $1611,1513,1458,1170,1063 .{ }^{1} \mathrm{H}$ NMR $\left(300 \mathrm{MHz}, \mathrm{CDCl}_{3}, \delta\right.$, ppm): 7.38-7.21 (m, 5H, - $\left.\mathrm{C}_{6} H_{5}\right), 4.77-4.69(\mathrm{~m}, 1 \mathrm{H},-\mathrm{OCH}), 4.60$ $\left(\mathrm{d}, 1 \mathrm{H}, J=11.6 \mathrm{~Hz},-\mathrm{OCH}_{2} \mathrm{Ph}\right), 4.45\left(\mathrm{~d}, 1 \mathrm{H}, J=11.6 \mathrm{~Hz},-\mathrm{OCH}_{2} \mathrm{Ph}\right)$, 3.76-3.66 (m, 1H, -OCH), 2.77-2.68 (m, 2H, $-\mathrm{CH}_{2}$ ), 1.88-1.79 (br. $\left.\mathrm{m}, 4 \mathrm{H}, 2 \mathrm{x}-\mathrm{CH}_{2}\right), 1.71-1.62\left(\mathrm{~m}, 2 \mathrm{H},-\mathrm{CH}_{2}\right), 1.31(\mathrm{~d}, 3 \mathrm{H}, J=6.0 \mathrm{~Hz}$, $-\mathrm{CH}_{3}$ ). ${ }^{13} \mathrm{C}$ NMR $\left(75 \mathrm{MHz}, \mathrm{CDCl}_{3}, \delta, \mathrm{ppm}\right): 172.8(1 \mathrm{C},-\mathrm{O}-\mathrm{C}=0$ ), $139.4(1 \mathrm{C}, \mathrm{Ar}-\mathrm{C}), 130.5$ (2C, $\mathrm{Ar}-\mathrm{C}), 129.5$ (1C, $\mathrm{Ar}-\mathrm{C}$ ), 128.1 (2C, $\mathrm{Ar}-\mathrm{C}$ ), 75.7 (1C, $-\mathrm{O}-\mathrm{CH}-), 72.3$ (1C, $\left.-\mathrm{O}-\mathrm{CH}_{2}-\right), 70.1$ (1C, $\left.-\mathrm{O}-\mathrm{CH}-\right)$, $38.7\left(1 \mathrm{C},-\mathrm{CH}_{2}-\right), 38.2\left(1 \mathrm{C},-\mathrm{CH}_{2}-\right), 33.3\left(1 \mathrm{C},-\mathrm{CH}_{2}-\right), 21.6(1 \mathrm{C}$, $\mathrm{CH}_{3}, 19.4\left(1 \mathrm{C},-\mathrm{CH}_{2}-\right)$. ESI-MS $(\mathrm{m} / \mathrm{z}): 271(\mathrm{M}+\mathrm{Na})^{+} .[\alpha]_{\mathrm{D}} 25:-75.4$ (c $0.16, \mathrm{CHCl}_{3}$ ). 


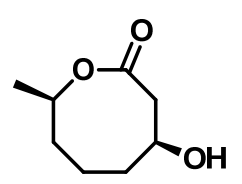

3

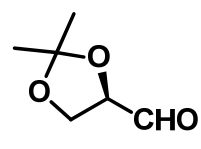

9

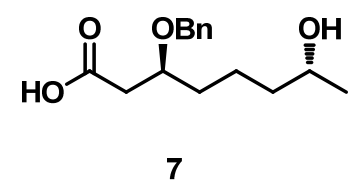

$\sqrt{ }$
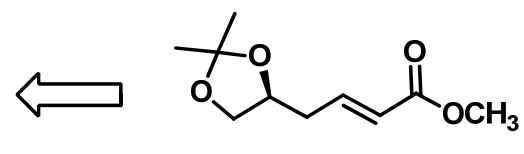

8

Scheme 2

\subsubsection{3. (-)Cephalosporolide D (3)}

To a solution of compound 19 ( $90 \mathrm{mg}, 0.32 \mathrm{mmol}$ ) in $\mathrm{CH}_{2} \mathrm{Cl}_{2}$ $(2 \mathrm{~mL}), \mathrm{TiCl}_{4}(0.02 \mathrm{~g}, 0.08 \mathrm{mmol})$ in $\mathrm{CH}_{2} \mathrm{Cl}_{2}(1 \mathrm{~mL})$ was added and it was allowed to stir at room temperature for $3 \mathrm{~h}$. After completion of the reaction, it was quenched with sat. $\mathrm{NaHCO}_{3}$ solution $(10 \mathrm{~mL})$, filtered and washed with $\mathrm{CH}_{2} \mathrm{Cl}_{2}(2 \times 10 \mathrm{~mL})$. The organic layer was dried over anhydrous $\mathrm{Na}_{2} \mathrm{SO}_{4}$ and evaporated under reduced pressure. Purification of the residue by column chromatography (60-120 Silica gel, 20\% EtOAc in petroleum ether) furnished compound 3 (Scheme 1).

(-) Cephalosporolide D (3): Color: Colorless syrup. Yield: 88\%. IR (Neat, $v, \mathrm{~cm}^{-1}$ ): 3422, 2931, 1721, 1646, 1439, 1283, 1159, 1118. ${ }^{1} \mathrm{H}$ NMR $\left(300 \mathrm{MHz}, \mathrm{CDCl}_{3}, \delta, \mathrm{ppm}\right): 4.67-4.60(\mathrm{~m}$, $1 \mathrm{H},-\mathrm{OCH}), 4.16-3.95(\mathrm{~m}, 1 \mathrm{H},-\mathrm{CH}), 2.92-2.61(\mathrm{dd}, 2 \mathrm{H}, J=5.8$, $11.9 \mathrm{~Hz}, 2 \mathrm{x}-\mathrm{CH}=\mathrm{CO}), 1.89-1.50\left(\mathrm{~m}, 8 \mathrm{H}, \mathrm{OH}, \mathrm{CH}, 3 \times \mathrm{CH}_{2}\right), 1.33$ $\left(\mathrm{d}, J=5.9 \mathrm{~Hz}, 3 \mathrm{H}, \mathrm{CH}_{3}\right) .{ }^{13} \mathrm{C} \mathrm{NMR}\left(75 \mathrm{MHz}, \mathrm{CDCl}_{3}, \delta, \mathrm{ppm}\right): 172.1$ $(1 \mathrm{C},-\mathrm{O}-\mathrm{C}=0), 75.1(1 \mathrm{C},-\mathrm{O}-\mathrm{CH}-), 71.4(1 \mathrm{C},-\mathrm{CH}-\mathrm{OH}), 43.3(1 \mathrm{C},-$ $\mathrm{CH}_{2}-$ ), $38.5\left(1 \mathrm{C},-\mathrm{CH}_{2}-\right), 36.6\left(1 \mathrm{C},-\mathrm{CH}_{2}-\right), 25.7\left(1 \mathrm{C},-\mathrm{CH}_{3}, 19.1\right.$ (1C, $-\mathrm{CH}_{2}-$ ). ESI-MS $(\mathrm{m} / \mathrm{z}): 181[\mathrm{M}+\mathrm{Na}]^{+} .[\alpha]_{\mathrm{D}} 25:-40.1\left(c 0.9, \mathrm{CHCl}_{3}\right)$.

\section{Results and discussion}

Our retrosynthetic analysis is outlined in Scheme 2. The macrolide 3 could be obtained by Yamaguchi lactonization followed by deprotection of benzyl ether of hydroxy acid $\mathbf{7}$. Compound $\mathbf{7}$, in turn, could be obtained from ester $\mathbf{8}$. The ester $\mathbf{8}$ could be prepared from $(R)$-glyceraldehyde derivative $\mathbf{9}$ by simple chemical transformations.

Accordingly, the known D-glyceraldehyde derivative 9, prepared from a literature procedure from D-mannitol [13] was subjected to Wittig olefination afforded ester 8 in $81 \%$ yield. Ester 8 was subjected to catalytic hydrogenation in the presence of $10 \% \mathrm{Pd}-\mathrm{C}$ in methanol under hydrogen atmosphere to give saturated ester $\mathbf{1 0}$ in $84 \%$. Later, ester $\mathbf{1 0}$ on further treatment with diisobutylaluminium hydride (DIBAL-H) in dry $\mathrm{CH}_{2} \mathrm{Cl}_{2}$ at $0{ }^{\circ} \mathrm{C}$ for $3 \mathrm{~h}$ gave corresponding aldehyde, which was subjected to $\alpha$-aminoxylation [14] catalyzed by L-proline, followed by in situ reduction using $\mathrm{NaBH}_{4}$ to furnish the required $\alpha$-amino-substituted diol, which upon reductive hydrogenation using $10 \% \mathrm{Pd} / \mathrm{C}$ in methanol afforded chiral diol 11 in $72 \%$ yield (>97\% diastereomeric excess). Diol 11 was treated with benzaldehyde dimethyl acetal in the presence of $p$ toluedine sulphonic acid (cat.) in dry $\mathrm{CH}_{2} \mathrm{Cl}_{2}$ at room temperature for $6 \mathrm{~h}$ to afford $\mathbf{1 2}$ in $83 \%$ yield. Regioselective ring opening of 12 with DIBAL-H (2M solution in toluene) at 0 ${ }^{\circ} \mathrm{C}$ to room temperature for $4 \mathrm{~h}$ afforded primary alcohol $\mathbf{1 3}$ in $79 \%$ yield.
The alcohol 13 on treatment with $\mathrm{I}_{2}$ in the presence of $\mathrm{PPh}_{3}$ and imidazole in dry THF afforded iodo-derivative 13a, which on subsequent treatment with homoallyl bromide and magnesium in THF at $0{ }^{\circ} \mathrm{C}$ for $8 \mathrm{~h}$ gave $\mathbf{1 4}$ in $83 \%$ yield, which was then subjected to epoxidation using $m$-chloroperbenzoic acid (m-CPBA) in dry $\mathrm{CH}_{2} \mathrm{Cl}_{2}$ at room temperature for $6 \mathrm{~h}$ to afford the racemic epoxide $\mathbf{1 5}$ in $\mathbf{7 0 \%}$ yield. Hydrolytic kinetic resolution of epoxide $\mathbf{1 5}$ using catalyst $(S, S)$-salen-Co-OAc catalyst in the presence of $\mathrm{AcOH}$ and $\mathrm{H}_{2} \mathrm{O}$ at $15{ }^{\circ} \mathrm{C}$ to room temperature for $12 \mathrm{~h}$ afforded $S$-epoxide 16 in 44\% yield [15]. Regioselective opening of epoxide $\mathbf{1 6}$ with lithium aluminium hydride in dry THF furnished alcohol $\mathbf{1 7}$ in 95\% yield. Hydrolysis of $\mathbf{1 7}$ with $60 \%$ aq. acetic acid at room temperature for $12 \mathrm{~h}$ gave diol 18, which on oxidative cleavage followed by oxidation with $\mathrm{NaClO}_{2}$ and $\mathrm{NaH}_{2} \mathrm{PO}_{4}, 2$-methyl-2-butene in aq. $t$ butanol afforded hydroxy acid $\mathbf{7}$ in $\mathbf{7 8 \%}$ yield. Hydroxy-acid $\mathbf{7}$ was subjected to macrolactonisation under Yamaguchi high dilution conditions using 2,4,6-trichlorobenzoyl chloride and $\mathrm{Et}_{3} \mathrm{~N}$ in dry THF to afford the lactone 19 in $64 \%$ yield [16]. Finally, 19 on debenzylation with $\mathrm{TiCl}_{4}$ in $\mathrm{CH}_{2} \mathrm{Cl}_{2}$ at room temperature for $3 \mathrm{~h}$ gave 3 in $88 \%$ yield. The ${ }^{1} \mathrm{H}$ NMR and ${ }^{13} \mathrm{C}$ NMR spectral data and optical rotation value of synthetic 3 were in good accord with those of the natural product (Scheme $1)$.

\section{Conclusion}

In conclusion, we have completed the total synthesis of the macrocyclic eight-membered ring lactone, (-)-Cephalosporolide $\mathrm{D}$ in a regioselective manner from $\mathrm{D}$-glyceraldehyde derivative. The desired stereochemistry was generated by $\alpha$-amino xylation catalyzed by L-proline reaction, Jacobsen's hydrolytic kinetic resolution.

\section{References}

[1]. Clemer, C.F.W.D. Pure Appl. Chem. 1971, 28, 413-454.

[2]. Keller-Schierlein, W. Fortschr. Chem. Org. Naturst. 1973, 30, 313-460.

[3]. Boeckman, J. R. K.; Fayos, J.; Clardy, J. J. Am. Chem. Soc. 1974, 96, 5954 5956.

[4]. Omura, O.; Nakagawa, A. J. Antibiotics 1975, 28, 401-433

[5]. Ackland, M. J.; Hanson, J. R.; Hitchcock, P. B.; Ratcliffe, A. H.; J. Chem. Soc., Perkin Trans. I 1985, 843-847.

[6]. Farooq, A.; Gordon, J.; Hanson, J. R.; Takahashi, J. A.; Phytochemistry 1995, 38, 557-558.

[7]. Shiina, I.; Fukuda, Y.; Ishii, T.; Fujisawa, H.; Mukaiyama, T. Chem. Lett. 1998, 8, 831-832.

[8]. Shiina, I.; Fujisawa, H; Ishii, T; Fukuda, Y.; Heterocycles 2000, 52 1105-1123.

[9]. Buszek, K. R.; Jeong, Y.; Sato, N; Still, P. C.; Muiño, P.L.; Ghosh, I.; Synth. Commun. 2001, 31, 1781-1781. 
[10]. Sabitha, G.; Reddy, T. R.; Ramesh, M.; Srinivas, C.; Yadav, J. S. Bull. Chem. Soc. Jpn. 2011, 84 (2), 229-231.

[11]. Reddy, G.V.; sreedhar, E.; Babu, K. S.; Rao, J. M. Tetrahedron Lett. 2010 , 51,1723-1726.

[12]. Shiina, I. Chem. Rev. 2007, 107, 239-273.

[13]. Bradshaw, J. S.; Huszthy, P.; McDaniel, C. W.; Zhu, C. Y.; Dalley, N. K.; Izatt, R. M. J. Org. Chem. 1990, 55, 3129-3137.

[14]. Hayashi, Y.; Yamaguchi, J.; Hibino, K.; Shoji, M. Tetrahedron Lett. 2003, 44, 8293-8296.

[15]. Tokunaga, M.; Larrow, J. F.; Kakiuchi, F.; Jacobsen, E. N. Science, 1997, 277, 936-938.

[16]. Inanga, J.; Hirata, K.; Saeki, H.; Katsuki, T.; Yamaguchi, M. Bull. Chem. Soc. Jpn. 1979, 52, 1989-1993. 